\title{
Pentaquarks and doubly heavy exotic mesons
}

\author{
Marek Karliner ${ }^{1, \star}$ \\ ${ }^{1}$ Raymond and Beverly Sackler School of Physics and Astronomy \\ Tel Aviv University, Tel Aviv, Israel
}

\begin{abstract}
I discuss the experimental evidence for and theoretical interpretation of the new mesons and baryons with two heavy quarks. These include doubly-heavy baryons, exotic hadronic quarkonia and most recently a manifestly exotic pentaquark-like doubly heavy baryon with a minimal quark content $u u d \bar{c}$ discovered by LHCb, whose mass, decay mode and width are in agreement with a prediction based on a physical picture of a deuteron-like $\Sigma_{c} \bar{D}^{*}$ "hadronic molecule".
\end{abstract}

\section{First observation of manifestly exotic hadrons}

In late 2007 the Belle Collaboration reported [1] anomalously large rate partial widths of $\Upsilon(5 S) \rightarrow$ $\Upsilon(2 S)$ and $\Upsilon(5 S) \rightarrow \Upsilon(1 S)$, two orders of magnitude larger than the analogous decays of $\Upsilon(3 S)$. Soon afterward Harry Lipkin and I proposed [2] that a four-quark exotic resonance $[b \bar{b} u \bar{d}]$ might mediate these decays through the cascade $\Upsilon(m S) \rightarrow[b \bar{b} u \bar{d}] \pi^{-} \rightarrow \Upsilon(n S) \pi^{+} \pi^{-}$. We suggested looking for the $[b \bar{b} u \bar{d}]$ resonance in these decays as peaks in the invariant mass of $\Upsilon(1 S) \pi$ or $\Upsilon(2 S) \pi$ systems. Subsequently Belle collaboration confirmed this prediction, with the observation $[3,4]$ of two charged bottomonium-like resonances $Z_{b}$ as narrow structures in $\pi^{ \pm} \Upsilon(n S)(n=1,2,3)$ and $\pi^{ \pm} h_{b}(m P)(m=1,2)$ mass spectra that are produced in association with a single charged pion in $\Upsilon(5 S)$ decays. The measured masses of the two structures averaged over the five final states are $M_{1}=10608.4 \pm 2.0 \mathrm{MeV}, M_{2}=10653.2 \pm 1.5 \mathrm{MeV}$, both with a width of about $15 \mathrm{MeV}$. Interestingly enough, the two resonances are only a few $\mathrm{MeV}$ away from the respective $B^{*} \bar{B}$ and $B^{*} \bar{B}^{*}$ thresholds.

This strongly suggests a parallel with $X(3872)$, whose mass is almost exactly at the $D^{*} \bar{D}$ threshold. It also raises the possibility that such states might have a complementary description as deuteron-like "molecule" of two heavy mesons quasi-bound by pion exchange [5-7].

The attraction due to $\pi$ exchange is 3 times weaker in the $I=1$ channel than in the $I=0$ channel. Consequently, in the charm system the $I=1$ state is expected to be well above the $D^{*} \bar{D}$ threshold and the $I=0 \quad X(3872)$ is at the threshold. ${ }^{1}$ In the bottom system the attraction due to $\pi$ exchange is essentially the same, but the kinetic energy is much smaller by a factor of $\sim m(B) / m(D) \approx 2.8$. Therefore the net binding in the bottom system is stronger than in the charm system.

This expectation has been confirmed by the discovery by BESIII [8] of charged exotic charmonium $Z_{c}(3900)$ at $3899.0 \pm 3.6 \pm 4.9 \mathrm{MeV}$ with $\Gamma=46 \pm 10 \pm 20 \mathrm{MeV}, 27 \mathrm{MeV}$ above $\bar{D} D^{*}$ threshold.

\footnotetext{
^e-mail:marek@proton.tau.ac.il

${ }^{1}$ For simplicity we treat $X(3872)$ as an isoscalar, since it has no charged partners, and we ignore here the issue of isospin breaking in its decays. A more refined treatment results in the same conclusions.
} 
The narrow widths of these exotic resonances implies that they are deuteron-like loosely bound states and/or near threshold resonances in the two heavy-meson system. Such "molecular" states, $\bar{D} D^{*}$, etc., were introduced in Ref. [5]. They were later extensively discussed $[6,7,9,10]$ in analogy with the deuteron which binds via exchange of pions and other light mesons, and were referred to as "deusons".

Since the quarks are heavy, we can treat their kinetic energy as a perturbation depending linearly on a parameter inversely proportional to $\mu_{\text {red }}$, the reduced mass of the two meson system, which scales like the mass of the heavy quark with the hamiltonian [11], $H=a \cdot p^{2}+V$, where $a=1 / \mu_{\text {red }} \sim 1 / m_{Q}$. We can use the existing data in order to make a very rough estimate of the isovector binding potential in the $m_{Q} \rightarrow \infty$ limit. We have two data points: $Z_{c}(3900) 27 \mathrm{MeV}$ above $\bar{D} D^{*}$ threshold and $Z_{b}(10610)$ approximately $3 \mathrm{MeV}$ above $\bar{B} B^{*}$ threshold. Linear extrapolation then yields approximately $-20 \mathrm{MeV}$ as the $\bar{B} B^{*}$ binding energy in the isoscalar channel.

The upshot is that the $Z_{c}(3900)$ isovector resonance confirms and refines the estimates in $[9,10]$ for the mass of the putative $\bar{B} B^{*}$ isoscalar bound state. This immediately lead to several predictions [11]: (a) two $I=0$ narrow resonances $X_{b}$ in the bottomonium system, about $20 \mathrm{MeV}$ below the corresponding $\bar{B} B^{*}$ and $\bar{B}^{*} B^{*}$ thresholds;

(b) an $I=1$ resonance above $\bar{D}^{*} D^{*}$ threshold;

(c) an $I=0$ resonance near $\bar{D}^{*} D^{*}$ threshold.

Soon after these predictions [11], the BESIII collaboration observed what looks just like (b) above, namely a new charmonium-like charged narrow resonance $Z_{c}(4025 / 4020)$, slightly above the $\bar{D}^{*} D^{*}$ threshold $[12,13]$. Fig. 1 provides a concise summary of the experimental information about the masses of doubly-heavy exotics observed so far, together with our predictions for additional states, as discussed above.

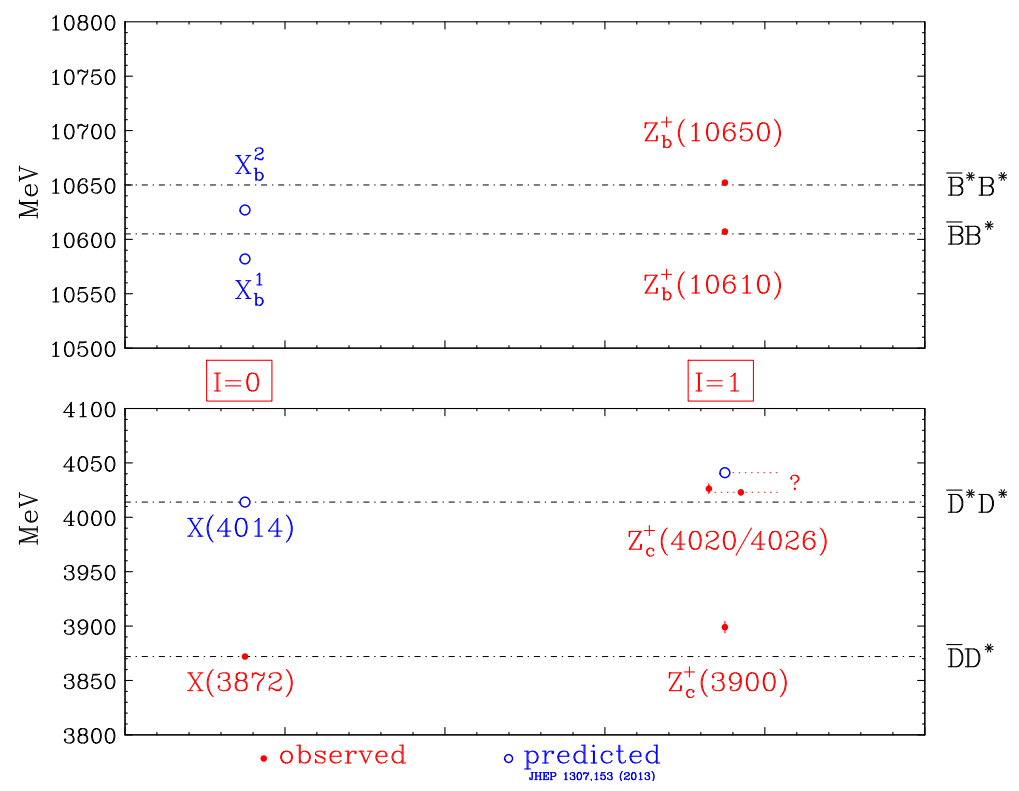

Figure 1. Masses of the doubly-heavy exotic quarkonia vs. two-meson thresholds. The states observed so far are shown in red, the predicted states are shown in blue. $I=0$ resonances are shown on the left and isovectors are shown on the right. Note the proximity of all the states to the corresponding two-meson thresholds. 
The $X_{b}$ states can most likely be observed through the decays $X_{b} \rightarrow \Upsilon \omega$ or $X_{b} \rightarrow \chi_{b} \pi \pi$. The decay to $\Upsilon \pi \pi$ is forbidden by $G$-parity conservation [14]. The seemingly analogous decay $X(3872) \rightarrow J / \pi \pi$ is only possible because isospin is strongly broken between $D^{+}$and $D^{0}$, and because $X(3872)$ is at the $\bar{D} D^{*}$ threshold. In contradistinction, in the bottomonium system isospin is almost perfectly conserved. Thus the null result in CMS search [15] for $X_{b} \rightarrow \Upsilon(1 S) \pi^{+} \pi^{-}$does not tell us if $X_{b}$ exists. In fact, $X_{b}$ might have been already observed by ATLAS, D0 and LHCb [16-19], camouflaging as run-of-the-mill excited bottomonium $\chi_{b}(3 P)[20]$.

When discussing molecular states (of which $X(3872)$ is at least a partial example), one notices that narrow resonances have been discovered very close to $\bar{D} D^{*}, \bar{D}^{*} D^{*}$ and $\bar{B} B^{*}, \bar{B}^{*} B^{*}$ thresholds, but no analogous resonances have been observed close to $\bar{D} D$ or $\bar{B} B$ threshold; cf. Table 1 . The absence of narrow resonances at $\bar{D} D$ and $\bar{B} B$ thresholds provides a strong hint that forces such as pion exchange play a role in the formation of these resonances, because one-pion exchange cannot bind two pseudoscalars. Moreover, the heavy-light mesons $D, D^{*}, B$ and $B^{*}$ contain only one light quark, so their coupling to pions is significantly smaller than $g_{\pi N N}$. Therefore in molecular systems containing two heavy-light mesons two-pion exchange is suppressed in comparison with one-pion exchange.

Strong support for the molecular interpretation of the states listed in Table 1 comes from the fact that they are quite narrow, despite many hundreds of $\mathrm{MeV}$ available for their decay into quarkonium and pion(s). The width is a product of the available (very large) phase space and the square of the absolute value of the matrix element between the initial and the final state. Thus the experiments tell us that the overlap of the resonance wave function with quarkonium must be very small. In a tightlybound pentaquark the the $c$ and $\bar{c}$ quarks are both within one confinement volume, so the overlap with the $J / \psi$ wave function is then generically large. On the other hand, in the molecular picture such a small overlap of the wave functions is automatic. This is because in a hadronic molecule the two heavy quarks spend most of their time far from each other. This point is schematically illustrated in Fig. 2.

Table 1. Five narrow exotic states close to meson-meson thresholds.

\begin{tabular}{ccccccc}
\hline \hline State & $\begin{array}{c}\mathrm{Mass}^{a} \\
(\mathrm{MeV})\end{array}$ & $\begin{array}{c}\mathrm{Width}^{a} \\
(\mathrm{MeV})\end{array}$ & $\begin{array}{c}\bar{Q} Q \\
\text { decay } \\
\text { mode }\end{array}$ & $\begin{array}{c}\text { Phase } \\
\text { space } \\
(\mathrm{MeV})\end{array}$ & $\begin{array}{c}\text { Nearby } \\
\text { threshold }\end{array}$ & $\begin{array}{c}\Delta E \\
(\mathrm{MeV})\end{array}$ \\
\hline$X(3872)$ & 3872 & $<1.2$ & $J / \psi \pi^{+} \pi^{-}$ & 495 & $\bar{D} D^{*}$ & $<1$ \\
$Z_{b}(10610)$ & 10608 & 21 & $\Upsilon \pi$ & 1008 & $\bar{B} B^{*}$ & $2 \pm 2$ \\
$Z_{b}(10650)$ & 10651 & 10 & $\Upsilon \pi$ & 1051 & $\bar{B}^{*} B^{*}$ & $2 \pm 2$ \\
$Z_{c}(3900)$ & 3900 & $24-46$ & $J / \psi \pi$ & 663 & $\bar{D} D^{*}$ & 24 \\
$Z_{c}(4020)$ & 4020 & $8-25$ & $J / \psi \pi$ & 783 & $\bar{D}^{*} D^{*}$ & 6 \\
\hline$\times$ & & & & & $\bar{D} D$ & \\
$\times$ & & & & & $\bar{B} B$ & \\
\hline
\end{tabular}

${ }^{a}$ Masses and widths approximate

${ }^{b}$ Quarkonium decay modes listed have maximum phase space

One then can infer from Table 1 the necessary conditions for existence of a near-threshold resonance of this type: 

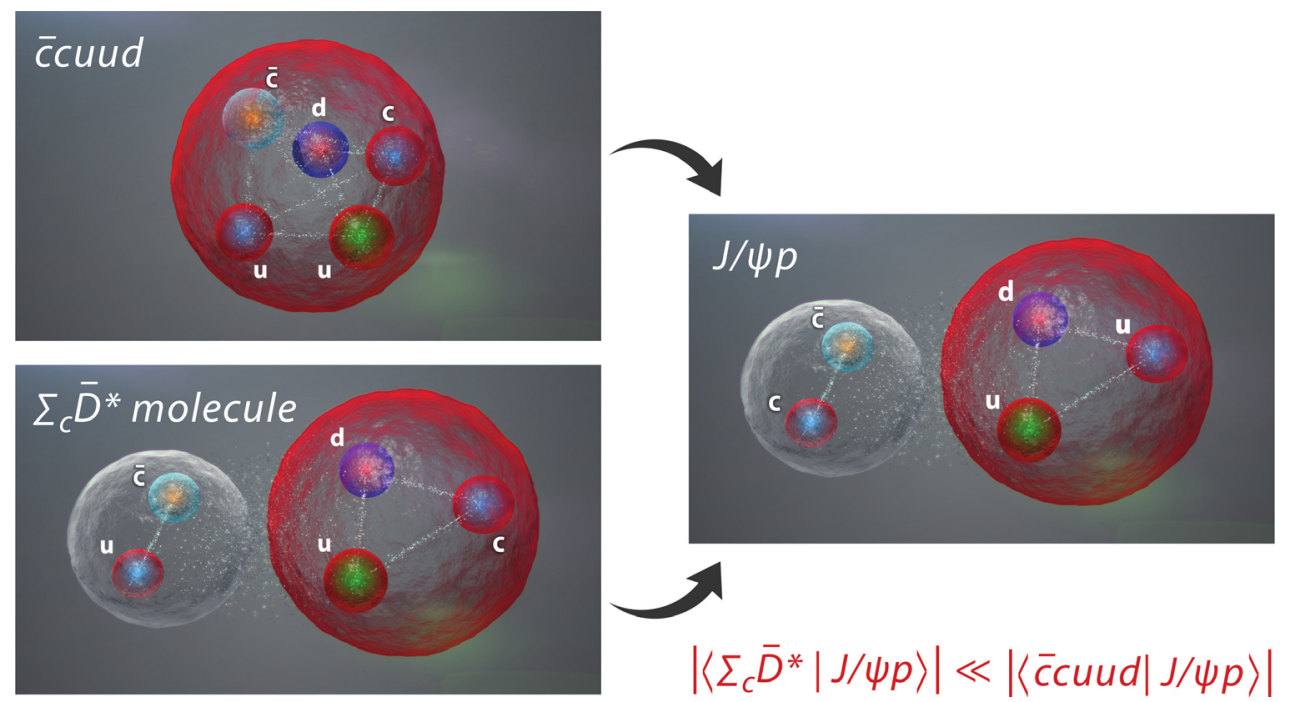

Figure 2. Schematic comparison of decay of a tightly-bound pentaquark vs. hadronic molecule into $J / \psi p$. The overlap of the hadronic molecule wave function with the $J / \psi p$ final state is generically much smaller than for a tightly-bound pentaquark.

(a) The state contains two heavy hadrons. They have to be heavy, as the repulsive kinetic energy is inversely proportional to the reduced mass.

(b) The two hadrons carry isospin, so that they can couple to pions. Channels like $\Sigma_{c} \bar{\Lambda}_{c}$, in which one of the particles has zero isospin, can exchange a pion to become the equal-mass channel $\Lambda_{c} \bar{\Sigma}_{c}$.

(c) The spin and parity of the two hadrons have to be such that they can bind through single pion exchange.

(d) The hadrons making up the molecule have to be sufficiently narrow, as the molecule's width cannot be smaller than the sum of its constituents' widths.

A crucial step is then the realization that the pion-exchange binding mechanism can in principle apply to any two heavy hadrons which satisfy conditions $(a)-(d)$ above, be they mesons or baryons.

A quantitative understanding of these effects then can be applied to other bound or resonant states of two heavy hadrons. For example, the discovery of the $Z_{b}$ and $Z_{c}$ states and their probable interpretation as $B^{*} \bar{B} / B^{*} \bar{B}^{*}$ and $D^{*} \bar{D} / D^{*} \bar{D}^{*}$ bound by pion exchange led us to propose that a weakly bound $\Sigma_{c} \bar{D}^{*}$ deuteron-like state might exist [21]. The narrow pentaquark resonance discovered by LHCb [22] has the right properties to be that state. Many more analogous loosely bound states of two heavy hadrons are predicted [21]. They are listed in Table 2. Note in particular the bottom quark analogue of the resonance discovered by LHCb [22], expected somewhat below the $\Sigma_{b} B^{*}$ threshold at $11140 \mathrm{MeV}$. Note also that in the open flavor sector we expect a $D^{*} B^{*}$ resonance, but no $D B^{*}$. This is because a bound state of a pseudoscalar and a vector of different flavors cannot bind by one pion exchange. The pentaquark states observed by LHCb in $\Lambda_{b}$ decay are excellent candidates for photoproduction off a proton target [23-25]. The size of the resonant cross sections is illustrated by Fig. 3 which shows the cross section for resonant photoproduction $\gamma p \rightarrow J / \psi p \rightarrow P_{c}(4450) \rightarrow J / \psi p$, as a function of the incident photon laboratory energy $E_{\gamma}$. The relevant experiments can be carried out at JLab. One of them has already been approved [26]. In principle, one can also search this way for the bottom analogue of $P_{c}$ (4450) [25]. 
Table 2. Thresholds for $Q \bar{Q}^{\prime}$ doubly-heavy molecular states

\begin{tabular}{ccccc}
\hline \hline Channel & $\begin{array}{c}\text { Minimum } \\
\text { isospin }\end{array}$ & $\begin{array}{c}\text { Minimal quark } \\
\text { content }\end{array}$ & $\begin{array}{c}\text { Threshold } \\
(\mathrm{MeV})^{c}\end{array}$ & $\begin{array}{c}\text { Example of } \\
\text { decay mode }\end{array}$ \\
\hline$D \bar{D}^{*}$ & 0 & $c \bar{c} q \bar{q}$ & 3875.8 & $J / \psi \pi \pi$ \\
$D^{*} \bar{D}^{*}$ & 0 & $c \bar{c} q \bar{q}$ & 4017.2 & $J / \psi \pi \pi$ \\
$D^{*} B^{*}$ & 0 & $c \bar{b} q \bar{q}$ & 7333.8 & $B_{c}^{+} \pi \pi$ \\
$\bar{B} B^{*}$ & 0 & $b \bar{b} q \bar{q}$ & 10604.6 & $\Upsilon(n S) \pi \pi$ \\
$\bar{B}^{*} B^{*}$ & 0 & $b \bar{b} q \bar{q}$ & 10650.4 & $\Upsilon(n S) \pi \pi$ \\
$\Sigma_{c} \bar{D}^{*}$ & $1 / 2$ & $c \bar{c} q q q^{\prime}$ & 4462.4 & $J / \psi p$ \\
$\Sigma_{c} B^{*}$ & $1 / 2$ & $c \bar{b} q q q^{\prime}$ & 7779.5 & $B_{c}^{+} p$ \\
$\Sigma_{b} \bar{D}^{*}$ & $1 / 2$ & $b \bar{c} q q q^{\prime}$ & 7823.0 & $B_{c}^{-} p$ \\
$\Sigma_{b} B^{*}$ & $1 / 2$ & $b \bar{b} q q q^{\prime}$ & 11139.6 & $\Upsilon(n S) p$ \\
$\Sigma_{c} \bar{\Lambda}_{c}$ & 1 & $c \bar{c} q q^{\prime} \bar{u} \bar{d}$ & 4740.3 & $J / \psi \pi$ \\
$\Sigma_{c} \bar{\Sigma}_{c}$ & 0 & $c \bar{c} q q^{\prime} \bar{q} \bar{q}^{\prime}$ & 4907.6 & $J / \psi \pi \pi$ \\
$\Sigma_{c} \bar{\Lambda}_{b}$ & 1 & $c \bar{b} q q^{\prime} \bar{u} \bar{d}$ & 8073.3 & $B_{c}^{+} \pi$ \\
$\Sigma_{b} \bar{\Lambda}_{c}$ & 1 & $b \bar{c} q q^{\prime} \bar{u} \bar{d}$ & $8100.9{ }^{d}$ & $B_{c}^{-} \pi$ \\
$\Sigma_{b} \bar{\Lambda}_{b}$ & 1 & $b \bar{b} q q^{\prime} \bar{u} \bar{d}$ & 11433.9 & $\Upsilon(n S) \pi$ \\
$\Sigma_{b} \bar{\Sigma}_{b}$ & 0 & $b \bar{b} q q^{\prime} \bar{q} \bar{q}^{\prime}$ & 11628.8 & $\Upsilon(n S) \pi \pi$ \\
\hline${ }^{a}$ Ignoring annihilation of quarks. & ${ }^{b}$ Plus other charge states when $I \neq 0$. & \\
${ }^{c}$ Based on isospin-averaged masses. & ${ }^{d}$ Thresholds differ by 27.6 MeV & \\
\hline \hline
\end{tabular}

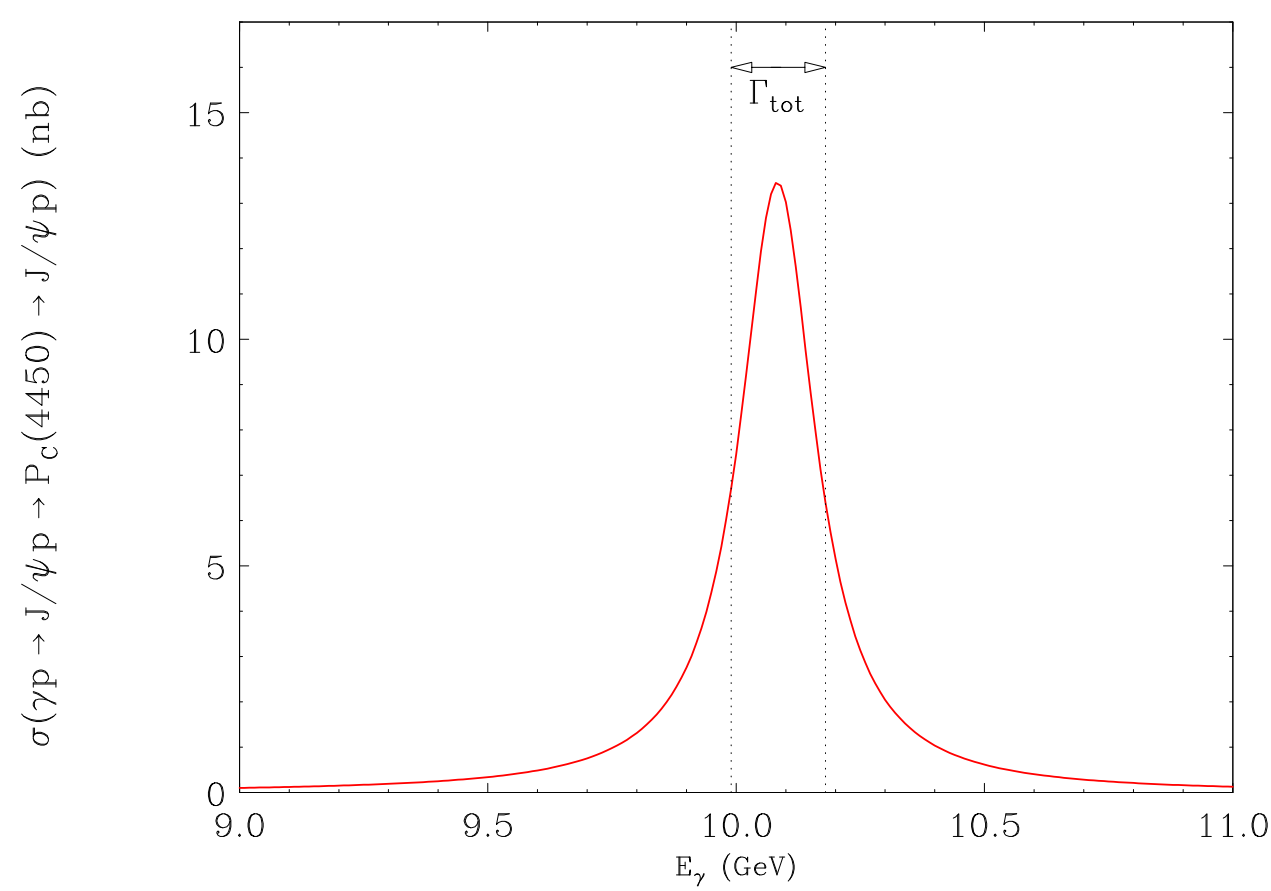

Figure 3. $\sigma\left(\gamma p \rightarrow J / \psi p \rightarrow P_{c}(4450) \rightarrow J / \psi p\right)$ resonant photoproduction cross section vs. the incident photon energy $E_{\gamma}$, assuming $B_{\text {out }}=0.1$ (see text). The width of the $P_{c}(4450)$ resonance is indicated by the vertical dotted lines. 
One can extend the molecular model to doubly heavy hadrons which do not contain light quarks, with binding provided by $\eta$ exchange [27], leading .e.g. to $D_{s} \bar{D}_{s}^{*}$ molecules, which might manifest themselves as $J / \psi \phi$ resonances, such as those recently observed by LHCb [28].

\section{A $\left(\Sigma_{b}^{+} \Sigma_{b}^{-}\right)$beauteron dibaryon?}

In addition to the states listed in Table 2, there is an interesting possibility of genuine di-baryons, e.g. a strongly bound $\Sigma_{b}^{+} \Sigma_{b}^{-}, \Sigma_{b}^{ \pm} \Sigma_{c}^{\mp}$ and $\Sigma_{c}^{+} \Sigma_{c}^{-}$deuteron-like states [9]. The $\Sigma_{b}$ is about $500 \mathrm{MeV}$ heavier than $B^{*}$. The $\Sigma_{b} \Sigma_{b}$ kinetic energy is therefore significantly smaller than that of $B \bar{B}^{*}$ or $B^{*} \bar{B}^{*}$. Moreover, $\Sigma_{b}$ with $I=1$ couples more strongly to pions than $B$ and $B^{*}$ with $I=\frac{1}{2}$. The opposite electric charges of $\Sigma_{b}^{+}$and $\Sigma_{b}^{-}$provide additional 2-3 MeV of binding energy. Analogous considerations apply to $\Sigma_{b}^{ \pm} \Sigma_{c}^{\mp}$ and $\Sigma_{c}^{+} \Sigma_{c}^{-}$states. The heavy dibaryon bound state might be sufficiently long-lived to be observed experimentally. A possible decay mode of the beauteron is $\left(\Sigma_{b}^{+} \Sigma_{b}^{-}\right) \rightarrow \Lambda_{b} \Lambda_{b} \pi^{+} \pi^{-}$, which might be observable in LHCb. It should also be seen in lattice QCD.

\section{References}

[1] K. F. Chen et al. [Belle Coll.], Phys. Rev. Lett. 100, 112001 (2008)

[2] M. Karliner and H. J. Lipkin, arXiv:0802.0649 [hep-ph]

[3] I. Adachi et al., Belle Coll., arXiv:1105.4583 [hep-ex]

[4] A. Bondar et al. [Belle Coll.], Phys. Rev. Lett. 108, 122001 (2012)

[5] M.B. Voloshin and L.B. Okun, JETP Lett. 23, 333 (1076) [Pisma Zh.Eksp.Teor.Fiz. 23, 369 (1976)]

[6] N. A. Tornqvist, Phys. Rev. Lett. 67, 556 (1991); Z. Phys. C 61, 525 (1994)

[7] N. A. Tornqvist, Phys. Lett. B 590, 209 (2004)

[8] M. Ablikim et al. [ BESIII Coll.], arXiv:1303.5949 [hep-ex]

[9] M. Karliner, H. J. Lipkin, N. A. Tornqvist, arXiv:1109.3472 [hep-ph]

[10] M. Karliner, H. J. Lipkin, N. A. Tornqvist, Nucl. Phys. Proc. Suppl. 225-227, 102 (2012)

[11] M. Karliner and S. Nussinov, JHEP 1307, 153 (2013)

[12] M. Ablikim et al. [BESIII Coll.], arXiv:1308.2760 [hep-ex]

[13] M. Ablikim et al. [BESIII Coll.], arXiv:1309.1896 [hep-ex]

[14] I thank Roman Mizuk for discussion on this point

[15] S. Chatrchyan et al. [CMS Coll.], arXiv:1309.0250 [hep-ex]

[16] G. Aad et al. (ATLAS Coll.), Phys. Rev. Lett. 108, 152001 (2012)

[17] V. M. Abazov et al. [D0 Coll.], Phys. Rev. D 86, 031103 (2012)

[18] R. Aaij et al. (LHCb Coll.), arXiv:1407.7734

[19] R. Aaij et al. (LHCb Coll.), arXiv:1409.1408

[20] M. Karliner and J. L. Rosner, Phys. Rev. D 91, 014014 (2015)

[21] M. Karliner and J. L. Rosner, Phys. Rev. Lett. 115, 122001 (2015)

[22] LHCb Coll., R. Aaij et al., Phys. Rev. Lett. 115, 072001 (2015)

[23] Q. Wang, X. H. Liu and Q. Zhao, Phys. Rev. D 92, 034022 (2015)

[24] V. Kubarovsky and M.B. Voloshin, Phys. Rev. D 92, 031502 (2015)

[25] M. Karliner and J. L. Rosner, Phys. Lett. B 752, 329 (2016)

[26] Z.-E. Meziani et al., arXiv:1609.00676 [hep-ex]

[27] M. Karliner and J. L. Rosner, Nucl. Phys. A 954, 365 (2016)

[28] R. Aaij et al. [LHCb Coll.], arXiv:1606.07895 [hep-ex] and arXiv:1606.07898 [hep-ex] 